\title{
Estimation of the Surface Velocity Field of the Aletsch Glacier Using Multibaseline Airborne SAR Interferometry
}

\author{
Pau Prats, Member, IEEE, Rolf Scheiber, Andreas Reigber, Member, IEEE, Christian Andres, and Ralf Horn
}

\begin{abstract}
This paper presents a methodology to process airborne interferometric synthetic aperture radar (SAR) data to measure surface velocity fields (SVFs) of temperate glaciers, and applies it to data acquired over the Aletsch glacier. The first part of this paper deals with the main limitation in airborne interferometric SAR to retrieve reliable interferometric products, namely, the existence of the so-called residual motion errors-inaccuracies on the order of a few centimeters in the navigation system. An extended multisquint approach is proposed for their estimation in the case of nonstationary scenes. The second part of this paper expounds an efficient methodology to derive SVFs with airborne systems, where the line-of-sight displacement is estimated using differential interferometry and the along-track component by estimating the azimuth coregistration offsets. The necessary steps to finally obtain the 3-D SVF are also presented, as well as the possibility of combining different acquisition geometries. Airborne interferometric SAR data acquired by the Experimental SAR system of the German Aerospace Center over the Aletsch glacier, located in the Swiss Alps, are used to evaluate the performance of the proposed approach. The motion of the corner reflectors deployed in the scene is retrieved with an accuracy between 1 and $5 \mathrm{~cm} /$ day using L-band data.
\end{abstract}

Index Terms-Cryosphere, differential synthetic aperture radar (SAR) interferometry (DInSAR), SAR interferometry.

\section{INTRODUCTION}

$\mathbf{T}$ HE MONITORING of alpine glaciers is essential to predict their evolution under the threat of global warming. However, data are often scarce (covering a few glaciers), given the difficulties in making field measurements in such high-altitude and remote scenarios. A solution is provided by remote-sensed synthetic aperture radar (SAR) data, which offers the possibility of obtaining surface velocity fields (SVFs) of glaciers within wide areas and with high accuracy. This information can be used within ice-flow models to derive the ice thickness of the glacier [1]. In particular, differential SAR interferometry (DInSAR) is a well-known technique to measure surface subsidence/motion for a wide range of applicationsmodeling surface deformation, landslides, soil compaction rate, atmosphere estimation, and glacier monitoring, among others. Spaceborne SAR systems have already proven the possibility

Manuscript received February 8, 2008; revised July 3, 2008. First published December 9, 2008; current version published January 28, 2009.

The authors are with the Microwaves and Radar Institute, German Aerospace Center, 82234 Oberpfaffenhofen, Germany (e-mail: Pau.Prats@dlr.de).

Digital Object Identifier 10.1109/TGRS.2008.2004277 to measure the SVFs [2]-[8]; however, the lack of flexibility in the acquisition configuration, as well as the fixed and usually too large revisit time, can become a limitation in many cases. Airborne SAR systems offer an excellent opportunity to overcome the limitations of spaceborne sensors, given their inherent flexibility in the sense of data-acquisition geometry and used wavelength, not to mention their higher spatial resolution. However, the processing of airborne data is not as straightforward as in the spaceborne case. The fact that the platform does not follow an ideal linear trajectory does not turn out to be a problem itself, considering that an efficient motion compensation (MoCo) is possible [9]-[11]. However, the main drawback is the existence of the so-called residual motion errors (RMEs) - inaccuracies in the navigation data on the order of a few centimeters. Such azimuth-varying errors can strongly limit the accuracy of the obtained interferograms, completely masking the motion to be measured.

This paper presents, for the first time, SVFs derived from airborne SAR interferometry. The first part of this paper (Section II) deals with the estimation of RMEs in nonstationary scenes. Among existing algorithms for estimating RMEs are those that estimate the difference between individual RME in an interferogram, i.e., the baseline error [12]-[15]. These approaches rely on the integration of the azimuth coregistration error between the interferometric images, considering that such offsets occur due to the presence of RMEs. Therefore, if the scene is experiencing some along-track motion, a biased estimation of the baseline error will result. Section II expounds this problem and proposes a solution based on an extension of the multisquint (MS) technique [15], which we have named extended MS (EMS).

The second part of this paper (Section III) is focused on the estimation of the SVFs of temperate glaciers using multibaseline airborne SAR interferometry. Once the baseline error is removed from the interferograms by using MS and EMS, it is possible to retrieve the 2-D SVFs in the azimuth/slant-range plane. In particular, conventional DInSAR is used to obtain the line-of-sight (LOS) displacement of the glacier, whereas the spectral diversity (SD) technique [16] is used to retrieve the along-track displacement. Note that an important advantage of using coherent techniques is that they do not rely on texture, hence working in areas with homogeneous backscattering. Section III discusses the necessary processing steps, including also the retrieval of the 3-D SVFs and the combination of different configurations. 
Finally, Section IV evaluates the performance of the proposed approach using airborne data acquired by the Experimental SAR (E-SAR) system of the German Aerospace Center (DLR) in the frame of the SWISAR campaign. The data were acquired at X-, C-, L-, and P-bands over the Aletsch glacier of the Swiss Alps in the years 2003 and 2006.

\section{EMS}

This section describes the retrieval of relative RMEs, i.e., baseline errors, in the presence of a nonstationary scene. As it will be shown, the effects of the RMEs and a real azimuth displacement in the scene between the acquisitions, from now on called a true coregistration error (TCE), are similar. Therefore, the separation of these two effects can become problematic. Section II-A explains the coupling between these two contributions, whereas Section II-B proposes an efficient solution for separating them on a pixel-by-pixel basis.

\section{A. Problem Statement}

Let us consider two interferometric signals, master and slave, where the master image is considered to be RME-free, while the slave image is affected by an RME, whose projection in LOS yields the following phase error:

$$
\begin{aligned}
\phi_{\mathrm{rme}}(t) & =\frac{4 \pi}{\lambda} \Delta r_{\mathrm{rme}}(t) \\
& \approx \frac{4 \pi}{\lambda} \cdot\left(a_{0}+a_{1} \cdot\left(t-t_{\mathrm{c}}\right)+\frac{a_{2}}{2} \cdot\left(t-t_{\mathrm{c}}\right)^{2}+\cdots\right)
\end{aligned}
$$

where $t$ is the azimuth time, $\lambda$ is the wavelength, $a_{i}$ represents the coefficients of the Taylor approximation around $t_{\mathrm{c}}$ of the error in the LOS $\Delta r_{\text {rme }}(t)$, and $t_{c}$ is the beam-center time instant, i.e., the time when the target is at the center of the beam

$$
t_{\mathrm{c}}=t_{0}-\frac{r_{0}}{v} \tan \beta
$$

where $t_{0}$ is the zero-Doppler time, $r_{0}$ is the closest approach distance, $v$ is the forward velocity, and $\beta$ is the processed squint angle. The 1-D compressed master signal in a zero-Doppler geometry using matched filtering is given by

$$
s_{\mathrm{m}}(t)=\operatorname{sinc}\left(B \cdot\left(t-t_{0}\right)\right) \cdot \exp \left[\mathbf{j} \phi_{\mathrm{m}}\right] \cdot \exp \left[\mathbf{j} 2 \pi f_{\mathrm{c}} \cdot\left(t-t_{0}\right)\right]
$$

where $\operatorname{sinc}(x)$ is the $\sin (\pi x) /(\pi x)$ function, $B$ is the signal bandwidth, $f_{\mathrm{c}}$ is the Doppler centroid, and $\phi_{\mathrm{m}}$ is a constant phase value. In the case of the slave signal, the linear component of the RME of (1) will introduce a shift in the impulse response given by [10], [17]

$$
\Delta t_{\mathrm{rme}}\left(t_{\mathrm{c}} ; L_{\mathrm{sa}}\right)=-\left.\frac{r_{0}}{v^{2}} \frac{\partial \Delta r_{\mathrm{rme}}(t)}{\partial t}\right|_{t=t_{\mathrm{c}}} \approx-\frac{r_{0} a_{1}}{v^{2}}
$$

where the dependence of $\Delta t_{\mathrm{rme}}$ on the synthetic aperture length $L_{\mathrm{sa}}$ is also stated. Aside from this shift, the slave signal is also affected by a coregistration error $\Delta t_{\text {tce }}$ due to the relative movement of the scene in the time interval between the acquisitions, where the subscript tce stands for the TCE. Consequently, the slave compressed signal is given by

$$
\begin{aligned}
s_{\mathrm{s}}(t)= & \operatorname{sinc}\left(B \cdot\left(t-t_{0}-\Delta t_{\mathrm{rme}}\left(t_{\mathrm{c}} ; L_{\mathrm{sa}}\right)-\Delta t_{\mathrm{tce}}\left(t_{0}\right)\right)\right) \\
& \cdot \exp \left[\mathbf{j} \phi_{\mathrm{s}}\right] \cdot \exp \left[\mathbf{j} \frac{4 \pi}{\lambda} a_{0}\right] \\
& \cdot \exp \left[\mathbf{j} 2 \pi f_{\mathrm{c}} \cdot\left(t-t_{0}-\Delta t_{\mathrm{rme}}\left(t_{\mathrm{c}} ; L_{\mathrm{sa}}\right)-\Delta t_{\mathrm{tce}}\left(t_{0}\right)\right)\right]
\end{aligned}
$$

where the effects of second and higher order terms have not been considered. The dependence of $\Delta t_{\text {rme }}$ with $t_{c}$ states that the induced shift due to the RMEs depends on the squint angle used to focus the signal, for a different squint angle implies a different time instant [see (2)] and, consequently, a different RME. Therefore, the final shift due to the RME will depend on the linear term of the RME along the processed aperture $L_{\mathrm{sa}}$ (processed bandwidth) for a given squint, or in other words, it will depend on the portion of the track used to focus the signal. On the other hand, $\Delta t_{\text {tce }}$ depends on the target position $t_{0}$ and, hence, is independent of both the processed squint and the processed aperture.

A suitable approach in estimating the coregistration error between two signals is the SD technique [16]. It consists of generating two low-resolution signals $s_{i}^{\mathrm{A}}$ and $s_{i}^{\mathrm{B}}$ by splitting the spectrum of the full-resolution one, where the superscripts A and B denote the different looks. By combining the master and slave looks in the following manner:

$$
s_{\mathrm{sd}}(t)=s_{\mathrm{m}}^{\mathrm{A}}(t) \cdot s_{\mathrm{s}}^{* \mathrm{~A}}(t) \cdot\left(s_{\mathrm{m}}^{\mathrm{B}}(t) \cdot s_{\mathrm{s}}^{* \mathrm{~B}}(t)\right)^{*}
$$

a differential interferogram is retrieved, whose phase information is related to the coregistration error with

$$
\Delta t(t)=\frac{\arg \left\{s_{\mathrm{sd}}(t)\right\}}{2 \pi \cdot\left(f_{\mathrm{c}}^{\mathrm{A}}-f_{\mathrm{c}}^{\mathrm{B}}\right)}
$$

where $f_{\mathrm{c}}^{\mathrm{A}}$ and $f_{\mathrm{c}}^{\mathrm{B}}$ are the central frequencies of the looks. Therefore, the coregistration error is estimated on a pixelby-pixel basis. Nevertheless, a filtering approach is usually performed to reduce the phase noise in the differential phase. The SD technique has been reported to achieve the Cramér-Rao bound for the estimation of the differential shift when the separation of the looks and their width are both equal to $1 / 3$ of the full bandwidth [18].

The technique presented in [13] exploits the possibility of estimating the baseline error using SD based on the fact that $\Delta t_{\mathrm{rme}}\left(t_{\mathrm{c}} ; L_{\mathrm{sa}}\right)$ depends on the squint angle used to focus the signal. The SD phase difference between two consecutive pixels will be slightly different, considering that their relative shift is $1 / \mathrm{PRF}$. Consequently, their $\Delta t_{\mathrm{rme}}\left(t_{\mathrm{c}} ; L_{\mathrm{sa}}\right)$ will depend on the linear terms of the RMEs along their corresponding synthetic apertures. Therefore, the measured coregistration error along the azimuth is indeed the derivative of the baseline error so that after a proper scaling and integration, the baseline error can be retrieved [13]-[15].

The MS technique [15] enhances the initial approach by using multiple looks instead of only two. It generates $N-1 \mathrm{SD}$ 
phases based on (6) from $N$ overlapped looks, which have a small width compared with the full-resolution bandwidth (for the E-SAR system, the look bandwidth is usually selected between 15 and $30 \mathrm{~Hz}$, considering that it depends on the characteristics of RMEs for a particular aircraft and navigation system). Afterward, the SD signals are aligned to the geometry of the track by applying a range-dependent shift given by

$$
\Delta t_{i}(r)=\frac{r}{v} \tan \bar{\beta}_{i, i+1}
$$

where $\beta_{i, i+1}$ is the mean squint between the two consecutive looks $i$ and $i+1$. This shift is necessary because the signal is focused in a zero-Doppler geometry; however, the location of the baseline error depends on the aspect angle used to focus the look. Finally, the differential phases are coherently added in order to reduce the phase noise. The reason for generating several looks is because of the behavior of the RMEs. If a large separation between looks is selected, then the RMEs are no longer linear through the two looks, resulting in an underestimation of the final baseline error, and similarly, a small look bandwidth ensures that the error is approximately linear. Considering that only two small looks would give a noisy result, several looks are generated instead, making use of the whole available bandwidth. Furthermore, an iterative approach is recommended in order to remove the underestimation.

In SD-based techniques for estimating RMEs, it is assumed that the only coregistration error in azimuth comes from the RMEs. This is usually a valid assumption when interferometric airborne data are processed with the same azimuth sampling and when parallel tracks are used. It ensures that the images are automatically aligned in the azimuthal dimension, but for a possible constant offset and the possible presence of the RMEs. However, if a localized TCE is present, the conventional approach will yield a biased estimation of the baseline error, hence degrading the final interferometric product. One might think of the possibility of masking the area where the motion is taking place, provided that it is known beforehand (which is the case in a glacier scenario). However, this option might result in insufficient data in areas under study where the motion area is large, as it is the case in the results shown in Section IV. In the following, an approach based on MS is proposed, which separates the two contributions on a pixel-by-pixel basis, hence allowing for a proper estimation of the baseline error, as in the case of a stationary scene.

\section{B. $E M S$}

The SD phase between the three equidistant looks A, B, and $\mathrm{C}$ can be written as

$$
\begin{aligned}
& d_{\mathrm{A}, \mathrm{B}}(t)=2 \pi \Delta f \cdot\left(\Delta t_{\mathrm{rme}}\left(t-\frac{r_{0}}{v} \tan \bar{\beta}_{\mathrm{A}, \mathrm{B}}\right)+\Delta t_{\mathrm{tce}}(t)\right) \\
& d_{\mathrm{B}, \mathrm{C}}(t)=2 \pi \Delta f \cdot\left(\Delta t_{\mathrm{rme}}\left(t-\frac{r_{0}}{v} \tan \bar{\beta}_{\mathrm{B}, \mathrm{C}}\right)+\Delta t_{\mathrm{tce}}(t)\right)
\end{aligned}
$$

where $\Delta f$ is the difference between the center frequencies. On the one hand, it can be observed that the contribution to

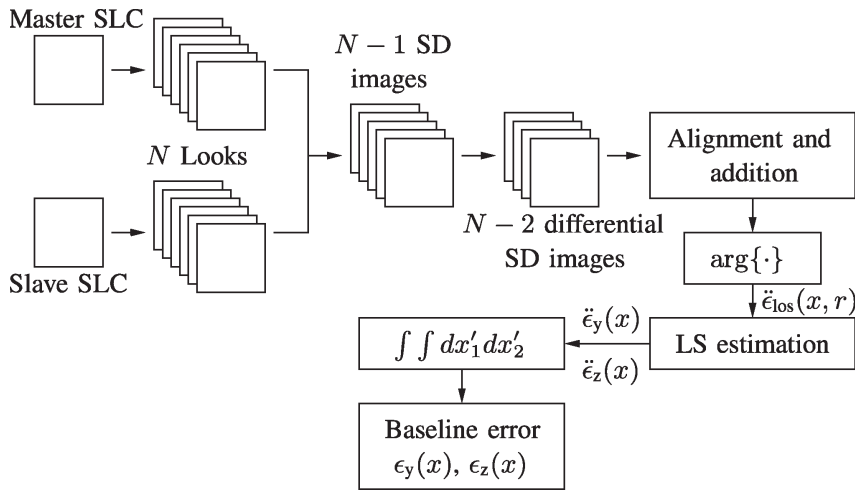

Fig. 1. Block diagram of the EMS approach. SLC stands for single-look complex and SD for spectral diversity.

the differential phase of the TCE $\Delta t_{\text {tce }}$ remains unchanged, independent of the look combination used. On the other hand, the vector $\Delta t_{\text {rme }}$ is shifted between the different looks an amount given by the distance between them. Hence, the $N-1$ SD phases can be written as:

$d_{i}(x)=\dot{f}_{\mathrm{be}}\left(x-r_{0} \tan \bar{\beta}_{i, i+1}\right)+g_{\mathrm{tce}}(x) \quad \forall i=1, \ldots, N-1$

where $\dot{f}_{\mathrm{be}}(\cdot)$ corresponds to the derivative of the baseline error (subscript be) and is related to $\Delta t_{\mathrm{rme}} ; g_{\mathrm{tce}}(\cdot)$ corresponds to the contribution of the TCE; and the space variable $x$ is used in substitution of the azimuth time $t$, for convenience. Note also that the range dependence has been omitted for simplicity.

Fig. 1 shows the proposed method to retrieve the baseline error without introducing a bias due to the TCE. From the $N$ looks, $N-1$ SD phases can be generated. The proposed solution, in order to separate the derivative of the baseline error from the TCE, is to take the difference between two adjacent SD phases. By doing so, the TCE given by $g_{\mathrm{tce}}(x)$ will be completely removed. Therefore

$$
\begin{aligned}
\dot{d}_{i}(x)= & \dot{f}_{\mathrm{be}}\left(x-r_{0} \tan \bar{\beta}_{i+1, i+2}\right) \\
& -\dot{f}_{\mathrm{be}}\left(x-r_{0} \tan \bar{\beta}_{i, i+1}\right) \quad \forall i=1, \ldots, N-2 .
\end{aligned}
$$

Moreover, if the squint centers are equispaced, it turns out that (11) is nothing but the second derivative of the baseline error, i.e.,

$$
\dot{d}_{i}(x) \approx \ddot{f}_{\mathrm{be}}\left(x-r_{0} \tan \bar{\beta}_{i}^{\prime}\right) \quad \forall i=1, \ldots, N-2
$$

where $\bar{\beta}_{i}^{\prime}$ is the mean squint between squints $\bar{\beta}_{i+1, i+2}$ and $\bar{\beta}_{i, i+1}$. This way, $N-2$ differential SD phases are retrieved. Similar to conventional MS, all differential SD phases need to be aligned to the same geometry, where the shift is now given by the mean angle $\bar{\beta}_{i}^{\prime}$. Each differential SD phase contains the same valuable information, i.e., the second derivative of the baseline error in the LOS $\ddot{\epsilon}_{\mathrm{los}}(x, r)$, and in order to reduce the phase noise, they are all added in the complex domain. Then, a model-based inversion (based on an least-squares (LS) solution), as presented in [15], is carried out to retrieve the second derivative of the horizontal $\ddot{\epsilon}_{\mathrm{y}}(x)$ and vertical $\ddot{\epsilon}_{\mathrm{z}}(x)$ 
baseline errors. Finally, a two-step integration yields the desired solution

$$
\epsilon_{i}=\int_{0}^{x_{1}^{\prime}} \int_{0}^{x_{2}^{\prime}} \frac{\ddot{\epsilon}_{i}}{\Delta x_{1} \cdot \Delta x_{2}} d x_{1}^{\prime} d x_{2}^{\prime}, \quad i=\{y, z\}
$$

with

$$
\begin{aligned}
& \Delta x_{1}=r_{0} \cdot\left(\tan \beta_{1}-\tan \beta_{0}\right) \\
& \Delta x_{2}=r_{0} \cdot\left(\tan \bar{\beta}_{1,2}-\tan \bar{\beta}_{0,1}\right)
\end{aligned}
$$

where $\Delta x_{1}$ is the distance corresponding to two consecutive looks and $\Delta x_{2}$ corresponds to the distance between the center frequencies of two SD phases.

One drawback of this approach is that aside from the constant and linear components of the baseline error, the quadratic component of the baseline error also remains unknown. Note that the original MS approach has the main limitation that constant and linear terms cannot be estimated. In [15], a solution is proposed to estimate them by means of an external digital elevation model (DEM) and an LS estimation. Similarly, the quadratic component can be added to the model to estimate it. The procedure consists in subtracting, from the interferometric phase, the synthetic phase computed with the DEM. Then, an LS estimation is applied to this residual phase to estimate the constant, linear, and quadratic terms of the baseline error. However, it has been found experimentally that with the E-SAR system, the quadratic component of the baseline error can be neglected for long data takes.

Due to the two-step integration, the EMS is more sensitive to phase noise compared with the conventional approach. The performance will mainly depend on the coherence of the scene; in practice, however, several iterations should be carried out to better estimate the baseline error. Once the baseline error is estimated, the track of the slave image can be updated with $\epsilon_{y}$ and $\epsilon_{z}$ so that both images will have the same RME after reprocessing, hence canceling out after the interfereogram generation. Finally, any azimuth coregistration error in the scene will correspond to a TCE, which can be efficiently estimated using the SD [16].

The main assumption made in the EMS approach is that the azimuthal coregistration error is not larger than the pixel resolution of a look. This usually applies for RMEs with the E-SAR system. However, this might not be the case with the azimuthal glacier movement so that special care must be taken in this case. Nevertheless, in the results shown in Section IV, the maximum azimuthal displacement of the glacier after one day is around one half of the azimuthal image resolution of the single-look complex for a processed bandwidth of $100 \mathrm{~Hz}$.

Concluding, the proposed EMS technique allows for the separation of RMEs and a TCE on a pixel-by-pixel basis based on the different nature of these two phenomena. Therefore, together with the LS estimation to retrieve the individual vertical and horizontal deviations, it turns into an efficient approach.

Further, less accurate approaches to separate the RME and the TCE based on their different natures are commented in [19].

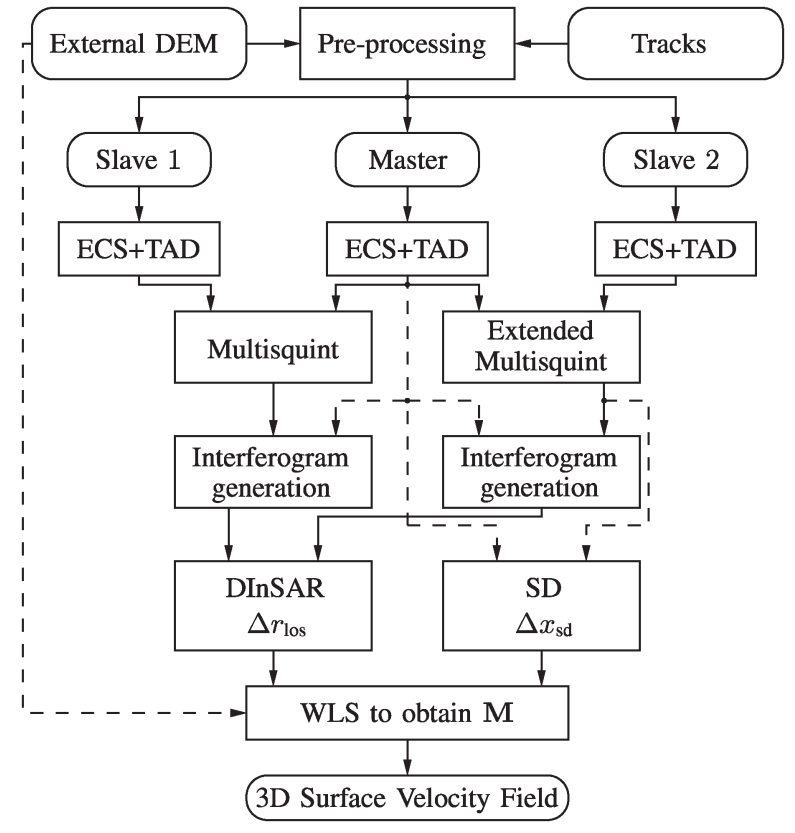

Fig. 2. Block diagram of the implemented processing chain to obtain the 3-D SVFs. ECS stands for extended chirp scaling, TAD for topography- and aperture-dependent (MoCo algorithm), SD for spectral diversity, and WLS for weighted least squares (estimation). $\mathbf{M}$ is the motion vector.

\section{Methodology to Retrieve SVFs With AIRBORNE INTERFEROMETRIC SAR}

The previous section dealt with the particularities of airborne systems in repeat-pass scenarios, where RMEs must be compensated. This section presents the methodology to retrieve the 3-D SVFs using multibaseline airborne SAR interferometry, as several steps must be carried out in order to retrieve reliable displacement information in the airborne case. In particular, the classical three-image DInSAR approach [20], [21] is used to retrieve the LOS displacement, where two images are acquired the same day and a third one (the long-term slave) is acquired after the event. In addition, the along-track displacement is retrieved by estimating the azimuth offsets between the master image and the long-term slave. In the present case, the SD technique is used [16]. Section III-A expounds the interferometric processing chain, whereas Section III-B details the procedure to obtain the 3-D SVFs.

\section{A. Interferometric SAR Processing}

Fig. 2 shows the proposed processing chain. The different steps are described next.

1) Preprocessing: In order to ease the forthcoming interferometric processing, several issues should be considered. On the one hand, the reference tracks should be parallel and with the same azimuth sampling for the three acquisitions. By doing so, the images will be automatically aligned along the azimuthal dimension, but for the existence of RMEs and TCE. On the other hand, the strong topography present in the scene must be considered during MoCo. As already discussed in several sources [10], [11], the assumption of a constant reference height, which is the standard approach when focusing SAR data using Fourier-based processing algorithms [9], [17], can 
introduce severe phase and azimuth coregistration errors in repeat-pass scenarios. Therefore, an external DEM is mandatory, taking into account that its accuracy and resolution will have a direct impact on the accuracy of the retrieved SVFs. There are several possibilities in order to take into account the topography, such as time-domain approaches [22] and topography- and aperture-dependent (TAD) MoCo algorithms [10], [11], [23]. With the use of the reference tracks, the external DEM is back-geocoded to the azimuth/slant-range plane, which is then used to compute the synthetic phase. The back-geocoded DEM is used by the TAD algorithm and for the computation of the local off-nadir angle, whereas the synthetic phase is used during the interferometric processing-range coregistration, coherence computation, and phase unwrapping.

2) SAR Processing: The raw data are focused using the extended chirp scaling (ECS) algorithm with the integrated two-step MoCo [9], whereas the subaperture topography- and aperture-dependent (SATA) algorithm [11] and the precise topography- and aperture-dependent (PTA) algorithm have been selected as TAD algorithms to accommodate the strong topography of the scene. In particular, SATA is used for the L-band data sets, whereas PTA is used with the P-band ones, as the resolution in the accommodation of the topography for SATA is degraded at lower frequencies [23].

3) Estimation of the Baseline Error: The conventional MS approach [15] is used with the short-term interferogram, whereas the EMS algorithm presented in Section II-B is used with the long-term one. Afterward, the slave tracks are updated with their corresponding baseline errors and are reprocessed. This way, all images have the same RME as the master image so that any combination of images will result in interferograms free of azimuth-varying baseline errors.

4) Interferometric Processing: A conventional interferometric processing is carried out. First, the range coregistration offsets computed from the external DEM are used to carry out the range interpolation for both slaves. The interferogram can now be generated for the short-term pair, as no TCE is present. However, SD is applied in both dimensions to estimate the TCE in the long-term interferogram. The retrieved offsets are used to further correct the coregistration values of the longterm slave. Finally, the long-term interferogram is generated, and a classical three-image DInSAR approach is performed in order to estimate the LOS displacement $\Delta r_{\text {los }}$. Together with the along-track displacement $\Delta x_{\mathrm{sd}}$ estimated before with SD, the 2-D SVFs of the glacier in the azimuth/slant-range plane is retrieved. It should be stressed that due to the azimuth-varying baseline of airborne systems, the real track deviations should be used to compute the height sensitivity, which is used to scale the residual interferometric phases during the DInSAR processing [24].

\section{B. Retrieval of the 3-D SVFs}

Once the 2-D SVF has been estimated, it is then possible to retrieve the 3-D one. To do so, it is necessary to make some assumptions, considering that for every pixel, only two measurements are available, which are $\Delta r_{\text {los }}$ and $\Delta x_{\mathrm{sd}}$; however, there are three unknowns for the displacement. This can be solved by assuming that the glacier flows in the direction of the maximum slope and that there is no emergence/submergence subsidence (surface parallel flow assumption) [6]. With the external DEM, it is possible to estimate the depression angle and orientation of the slope, yielding the direction of the movement, so that only the magnitude of the displacement $M$ is unknown. Considering that, now, two measurements are available with only one unknown, a weighted LS (WLS) estimation to retrieve the magnitude of the displacement $M$ can be performed using

$$
M=\left(\mathbf{H}^{\mathrm{T}} \mathbf{W} \mathbf{H}\right)^{-1} \mathbf{H}^{\mathrm{T}} \mathbf{W} \boldsymbol{\Delta}
$$

with

$$
\begin{aligned}
\mathbf{H} & =\left[\begin{array}{l}
\hat{\mathbf{e}}_{\mathrm{M}} \cdot \hat{\mathbf{e}}_{\mathrm{los}} \\
\hat{\mathbf{e}}_{\mathrm{M}} \cdot \hat{\mathbf{e}}_{\mathrm{sd}}
\end{array}\right] \\
\boldsymbol{\Delta} & =\left[\begin{array}{c}
\Delta r_{\mathrm{los}} \\
\Delta x_{\mathrm{sd}}
\end{array}\right] \\
\mathbf{W} & =\left[\begin{array}{cc}
1 / \sigma_{\mathrm{los}}^{2} & 0 \\
0 & 1 / \sigma_{\mathrm{sd}}^{2}
\end{array}\right]
\end{aligned}
$$

where $\hat{\mathbf{e}}_{\mathrm{M}}$ is the unitary vector indicating the direction of the maximum slope, $\hat{\mathbf{e}}_{\text {los }}$ indicates the LOS direction, $\hat{\mathbf{e}}_{\text {sd }}$ indicates the along-track direction of the sensor, $\sigma_{\text {los }}$ and $\sigma_{\mathrm{sd}}$ are the standard deviations of the LOS and along-track measurements, respectively, and the symbol - stands for the dot product. Note that, in this case, the computation of the inverse is trivial, as the term $\mathbf{H}^{\mathrm{T}} \mathbf{W H}$ is a scalar. The phase standard deviations can be retrieved using

$$
\begin{aligned}
\sigma_{\text {los }} & =\frac{\lambda}{4 \pi} \sqrt{\sigma_{\phi_{\text {long }}}^{2}+\frac{B_{\perp, \text { long }}^{2}}{B_{\perp, \text { short }}^{2}} \sigma_{\phi_{\text {short }}}^{2}} \\
\sigma_{\text {sd }} & =\frac{3 \sqrt{3}}{4 \sqrt{L_{\mathrm{sd}}}} \frac{\sqrt{1-\gamma_{\text {long }}^{2}}}{\pi \gamma_{\text {long }}} \frac{v}{\text { PRF }}
\end{aligned}
$$

where the subscripts short and long refer to the short- and long-term interferograms, respectively, $L_{\mathrm{sd}}$ is the number of independent looks used to reduce the noise in the SD phase, $\gamma$ is the interferometric coherence, PRF is the pulse repetition frequency, and $B_{\perp}$ is the perpendicular baseline. The formula to compute $\sigma_{\mathrm{sd}}$ is given in [18], where the separation and width of the looks for the SD have been selected to be $1 / 3$ of the processed bandwidth. The phase standard deviation $\sigma_{\phi}$ can be computed using the well-known formula given in [25]

$$
\sigma_{\phi_{i}}=\frac{1}{\gamma_{i}} \sqrt{\frac{1-\gamma_{i}^{2}}{2 L_{\operatorname{los}_{i}}}}, \quad i=\{\text { short, long }\}
$$

where $L_{\mathrm{los}}$ is the number of looks applied to the interferometric phase. Fig. 3 shows the accuracy in the measurement of the LOS and along-track motions for a different number of looks. The L-band parameters shown in Table II have been used to generate the plots, with a ratio between perpendicular baselines equal to 0.1. As expected, the accuracy of the LOS displacement is more than one order of magnitude more accurate than the along-track one for an equal number of looks.

A last step involves converting the retrieved 3-D SVF, which is in a local coordinate frame (with the $x$-axis pointing to 

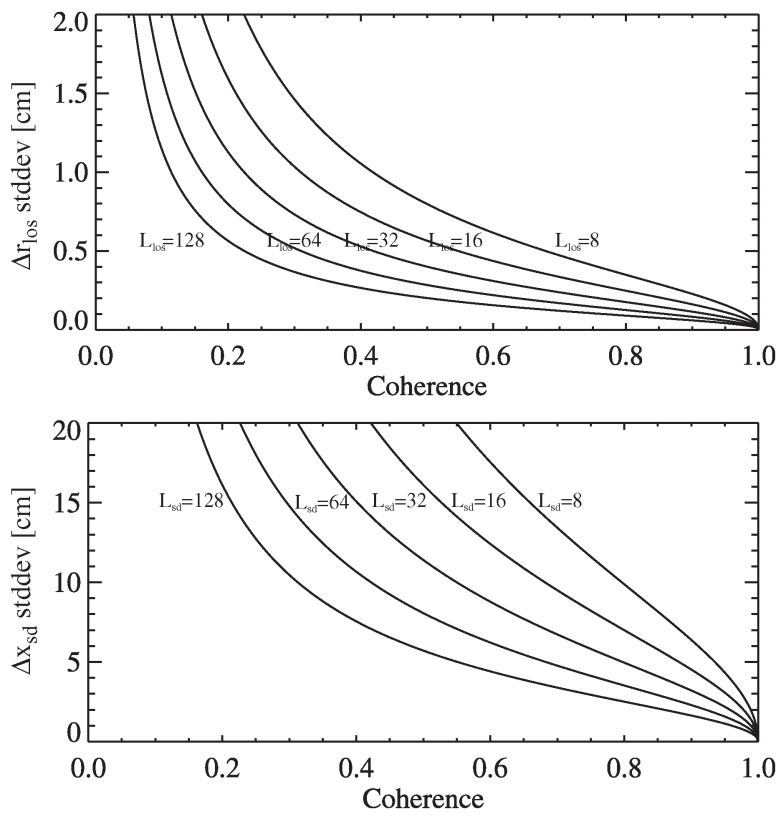

Fig. 3. Standard deviation of the estimation for (top) $\Delta r_{\text {los }}$ and (bottom) $\Delta x_{\mathrm{sd}}$ for different number of looks. The LOS estimation is almost one order of magnitude more accurate than the SD one for the same number of looks.

the along-track direction), to a global reference system, e.g., WGS-84 or Universal Transverse Mercator (UTM). It is worth commenting that the intrinsic flexibility of an airborne platform allows multiple acquisitions in a short time frame. Therefore, several tracks can be flown in order to retrieve different projections of the displacement and consequently obtain a better estimation of the SVF [4]. In fact, with one more pair of measurements, e.g., acquired in a track perpendicular to the previous one, it is possible to avoid any assumption concerning the direction of the glacier motion, hence estimating directly the motion vector $\mathbf{M}[8]$. The system of equations, in that case, would be

$$
\left[\begin{array}{c}
\hat{\mathbf{e}}_{1} \\
\vdots \\
\hat{\mathbf{e}}_{i}
\end{array}\right]\left[\begin{array}{c}
M_{\mathrm{x}} \\
M_{\mathrm{y}} \\
M_{\mathrm{z}}
\end{array}\right]=\left[\begin{array}{c}
\Delta_{1} \\
\vdots \\
\Delta_{i}
\end{array}\right], \quad i \geq 3
$$

where the subscript $i$ indicates different measurements either in LOS or along-track. The solution can be retrieved again via WLS. However, it will be necessary to transform all unitary vectors of the different slant-range geometries to the same coordinate system. Section IV shows an example using two DInSAR and two SD measurements obtained with two perpendicular configurations.

As a final comment, note that because two measurements are available, an altenative approach for (16) would be to project the displacements on the plane defined by the slope, instead of using the slope vector, so that the $x$ and $y$ components of the velocity are retrieved while the $z$ component is constrained by the slope [4].

\section{EXPERIMENTAL RESULTS}

Data acquired by DLR's E-SAR system are used to validate the proposed approach. The data were acquired over the Aletsch
TABLE I

E-SAR FLIGHTS WITHIN THE SWISAR CAMPAIGN

\begin{tabular}{|c|c|c|c|c|}
\hline Flight & Band & Baseline & Date\&Local Time & Configuration \\
\hline$\overline{0305}$ & $\mathrm{~L}$ & - & 10/27/2003 12:02 P.M. & FISA \\
\hline 0306 & $\mathrm{~L}$ & $11 \mathrm{~m}$ & 10/27/2003 12:13 P.M. & FISA \\
\hline 0401 & $\mathrm{~L}$ & $0 \mathrm{~m}$ & 10/28/2003 11:36 A.M. & FISA \\
\hline 0805 & $\mathrm{~L}$ & - & 10/17/2006 12:21 P.M. & FISA \\
\hline 0806 & $\mathrm{~L}$ & $7 \mathrm{~m}$ & 10/17/2006 12:35 P.M. & FISA \\
\hline 0702 & $\mathrm{~L}$ & $0 \mathrm{~m}$ & 10/16/2006 12:19 P.M. & FISA \\
\hline 0706 & $\mathrm{P}$ & - & 10/16/2006 1:03 P.M. & FISA \\
\hline 0808 & $\mathrm{P}$ & $0 \mathrm{~m}$ & 10/17/2006 12:57 P.M. & FISA \\
\hline 0701 & $\mathrm{~L}$ & - & 10/16/2006 12:09 Р.M. & JUJO \\
\hline 0703 & $\mathrm{~L}$ & $7 \mathrm{~m}$ & 10/16/2006 12:30 P.M. & JUJO \\
\hline 0801 & $\mathrm{~L}$ & $0 \mathrm{~m}$ & 10/17/2006 11:40 A.M. & JUJO \\
\hline$\overline{0705}$ & $P$ & - & 10/16/2006 12:52 P.M. & JUJO \\
\hline 0807 & $\mathrm{P}$ & $0 \mathrm{~m}$ & 10/17/2006 12:48 P.M. & JUJO \\
\hline
\end{tabular}

TABLE II

Main System ANd Processing Parameters (L-/P-BAndS)

\begin{tabular}{lc}
\hline System $P R F$ & $400 \mathrm{~Hz} / 500 \mathrm{~Hz}$ \\
Sampling frequency & $100 \mathrm{MHz} / 100 \mathrm{MHz}$ \\
Processed azimuth bandwidth & $100 \mathrm{~Hz} / 125 \mathrm{~Hz}$ \\
Processed range bandwidth & $94 \mathrm{MHz} / 70 \mathrm{MHz}$ \\
Wavelength & $23 \mathrm{~cm} / 85 \mathrm{~cm}$ \\
Mid range & $4000 \mathrm{~m}$ \\
Mean flight altitude above ground & $2800 \mathrm{~m}$ \\
Mean forward velocity & $90 \mathrm{~m} / \mathrm{s}$ \\
Processing squint angle & $0^{\circ}$ \\
Polarization & $\mathrm{VV}$ \\
\hline
\end{tabular}

glacier (located in the Swiss Alps), in the frame of the SWISAR campaign, in the years 2003 and 2006. Several data takes were performed at P-, L-, C-, and X-band; however, only P- and L-band were acquired on two consecutive days in order to perform differential interferometry. Table I summarizes the acquisitions, whereas Table II shows the main system and processing parameters for P- and L-band. The flight altitude above mean sea level was around $6000 \mathrm{~m}$, and considering that the aircraft, a Dornier 228, is not pressurized, the crew had to wear oxygen masks during the flights. The configuration acronyms stand for Fieschersattel (FISA) and Jungfraujoch (JUJO), named after two geographical locations in the area.

Fig. 4 shows the flight configuration, where it can be noted that the FISA configuration was acquired from opposite flights in 2003 and 2006. Note also that there are only two P-band acquisitions for every configuration so that, in this case, the external DEM is used to remove the topography when performing DInSAR. It is worth mentioning that although the long-term flights were acquired with a mean baseline of $0 \mathrm{~m}$, motion deviations from the reference track introduce a small azimuthvarying baseline, hence being sensitive to the topography and requiring a third image or a DEM to remove it.

Section IV-A shows results to validate the EMS technique with one of the L-band data takes, whereas Section IV-B focuses on the retrieval of the SVF of the Aletsch glacier.

\section{A. EMS Validation}

This section presents results to show the performance of the proposed EMS approach with L-band data corresponding to the 2003 campaign. In particular, the baseline error was computed between acquisitions 0305 and 0401, i.e., with a one-day 


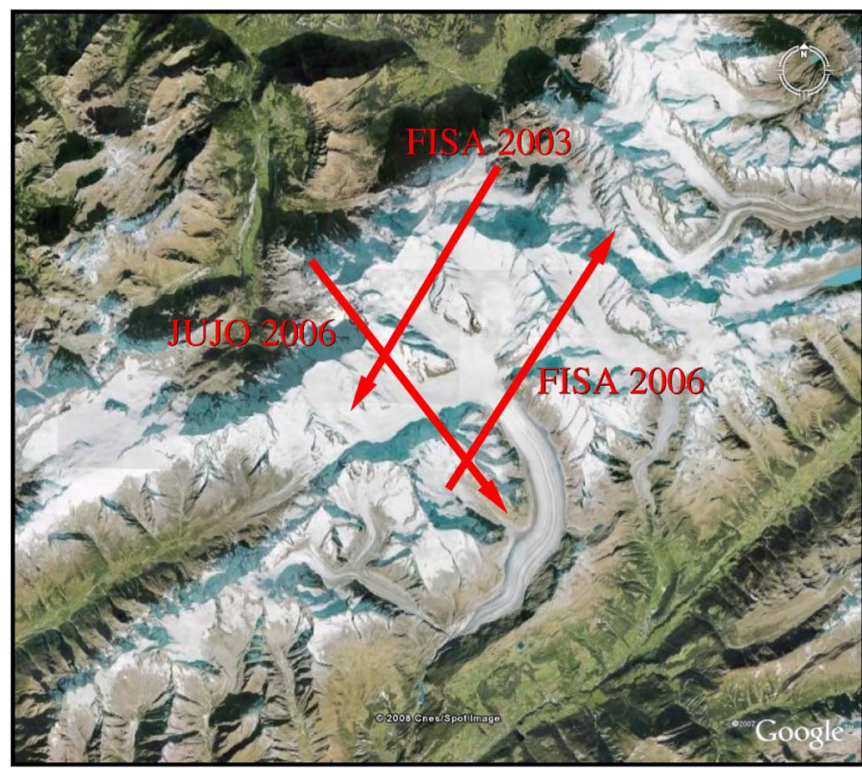

Fig. 4. Flight configuration for 2003 and 2006 campaigns. Both the P- and L-band antennas are left-looking.
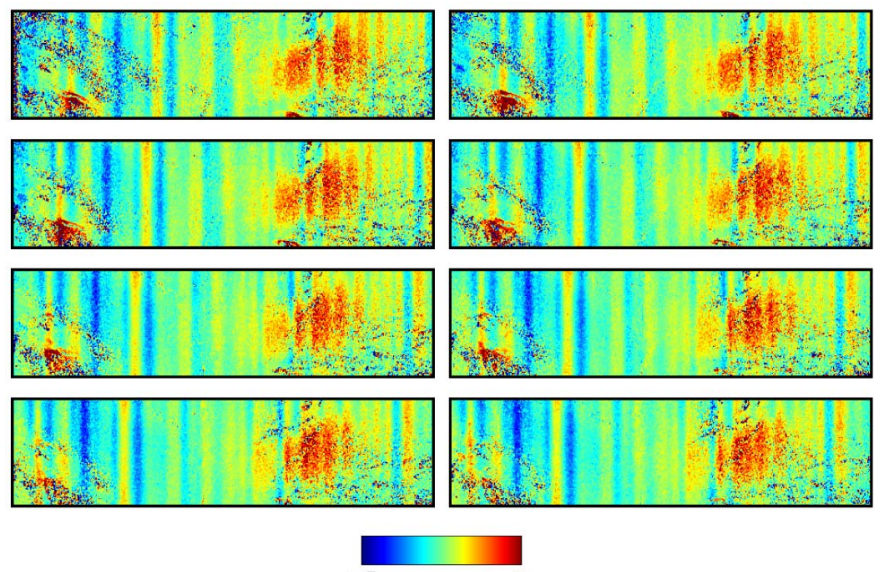

Fig. 5. Spectral diversity phases of different looks between two images where a TCE is present. The vertical stripes correspond to the derivative of the baseline error, whereas the large red patch on the right side of the image corresponds to an area experiencing a TCE. The azimuth direction is horizontal and the range one is vertical, with near range on top of the images.

difference in order to have a TCE. The number of generated looks was $N=9$, with a bandwidth of $30 \mathrm{~Hz}$ each and with center frequencies equal to $-60,-45,-30,-15,0,15,30,45$, and $60 \mathrm{~Hz}$ (note that, in this case, the processed bandwidth was $200 \mathrm{~Hz}$ to allow for a better performance). The resulting number of SD images is therefore eight, and their phases are shown in Fig. 5. First of all, one can notice the vertical stripes, which correspond to the baseline error, and how they shift in azimuth between consecutive looks. However, the interesting effect to note is the big red patch on the right side of the image, which can be observed in all of them. This patch corresponds, indeed, to a TCE, and it does not move for different SD phases. On the left side of the image, there is also some area experiencing a TCE (blue area); however, in this case, it is more difficult to see, considering that the TCE is smaller. If these SD images are aligned and added to estimate the baseline error, a bias will be
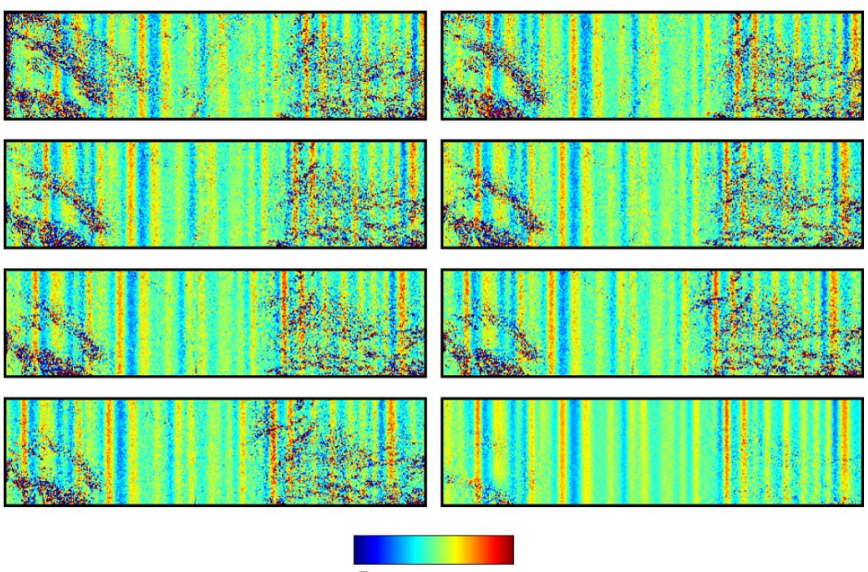

Fig. 6. Differential SD phases of the SD phases shown in Fig. 5. In this case, the vertical stripes correspond to the second derivative of the baseline error, whereas the contribution due to the TCE has been canceled out. (Bottom right) Coherent addition of the differential SD phases after aligning them to the track geometry (beam-center geometry), which helps in reducing the phase noise. The azimuth direction is horizontal and the range one is vertical, with near range on top of the images.

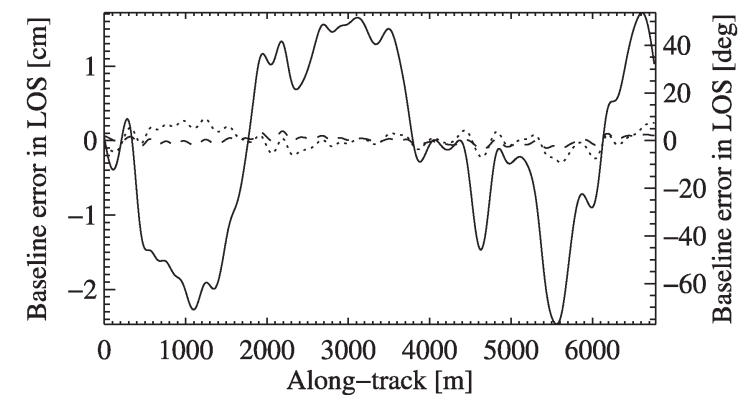

Fig. 7. Estimated baseline error using the EMS at mid range for the (solid) first, (dotted) second, and (dashed) third iterations. FISA configuration, L-band, 2003 campaign.

introduced due to the undesired effect of a TCE. Therefore, the solution proposed in this paper is to use EMS by just making the difference between two consecutive SD phases. Consequently, the TCE contribution cancels out, as it remains constant among looks. Fig. 6 shows this effect by showing the seven differential SD phases. These phases correspond to the second derivative of the baseline error, and note how the effect due to the TCE is no longer visible. The drawback is that the noise level has increased; however, it is effectively reduced when all phases are aligned and added, as shown in the bottom right image of Fig. 6. Finally, the baseline error is retrieved after applying the LS estimation to the differential SD phase and integrating twice as expressed in (13). Fig. 7 shows the estimated baseline error in LOS at midrange. The result after the second and third iterations is also shown, proving that the baseline error can be properly estimated and removed. Correcting the baseline error ensures that any remaining coregistration error comes from a TCE and, also, the phase accuracy is improved.

\section{B. SVF of the Aletsch Glacier}

Fig. 8 shows the reflectivity image at L-band for the 2006 acquisition, whereas Fig. 9 shows the external X-band DEM 


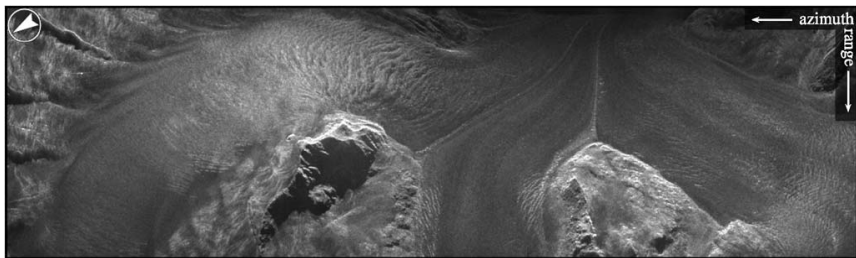

Fig. 8. L-band reflectivity image for the 2006 campaign, FISA configuration.

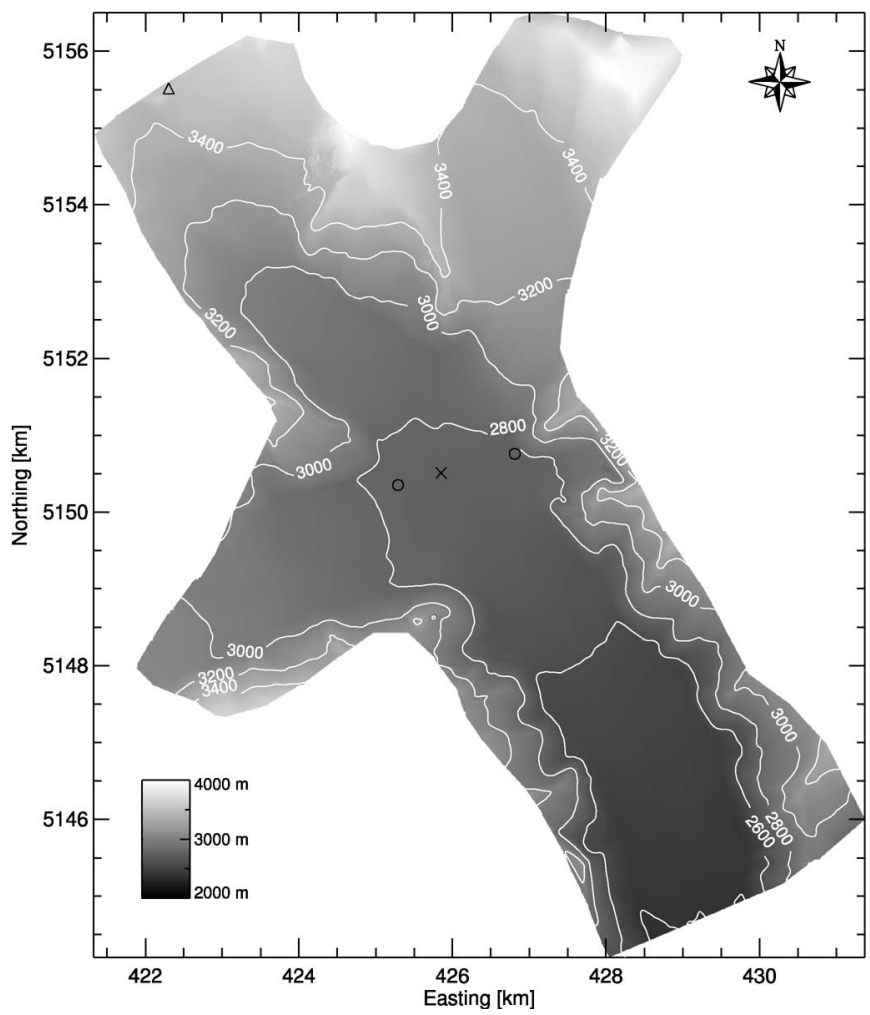

Fig. 9. X-band DEM used during MoCo for the 2006 campaign. The cross and the black circles indicate the location of the CRs in the 2003 and 2006 campaigns, respectively. The black triangle indicates the location of the scientific observatory located at Jungfraujoch.

in a UTM projection. This DEM was computed from singlepass interferometric SAR data acquired by the E-SAR system during one of the two days of the campaign. Note that two DEMs were generated, one for each year, considering that large changes can occur even in a few months' difference due to the fast glacier dynamics. In order to improve the quality of this DEM, the X-band data were reprocessed with a TAD algorithm using the DEM of the first iteration. Then, after processing an L-band short-term interferogram, an L-band DEM was retrieved, which was used to reprocess the data. By doing so ensures that the heights of the DEM correspond to an L-band phase center rather than to the X-band one, improving, this way, the performance of the MoCo step and avoiding undesired azimuth shifts due to unknown topography [24]. Note that this approach was not possible with the P-band, as there were no short-term acquisitions available. Nevertheless, the P-band data were processed using the L-band DEM.

The calibration of the interferometric phase before applying DInSAR is a major issue in airborne SAR. Usually, the largest error that remains after applying MS is a constant baseline
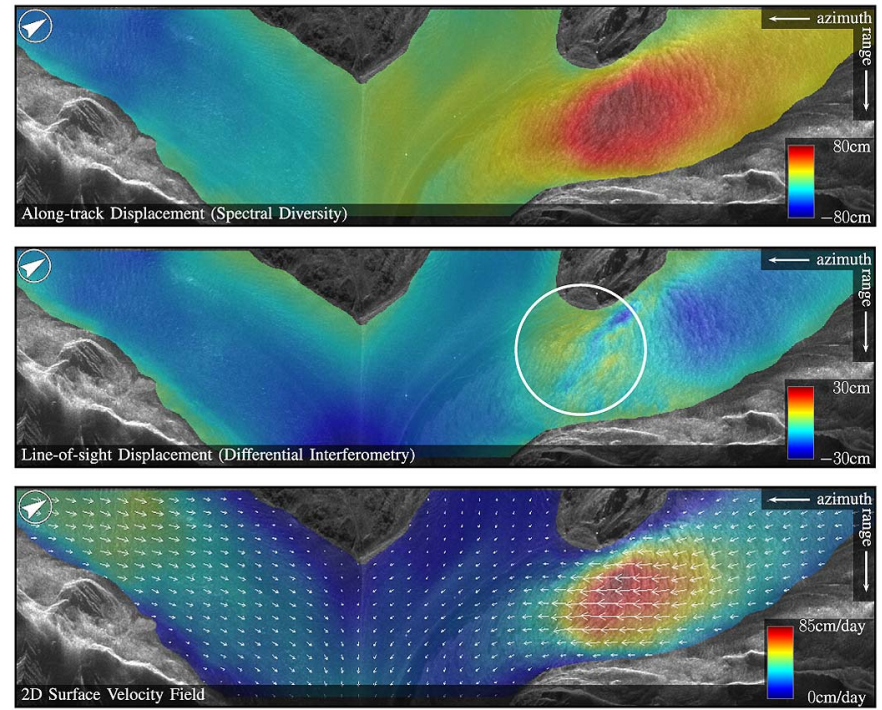

Fig. 10. Retrieval of the 2-D SVFs. (Top) Along-track displacement measured with SD. (Middle) LOS displacement measured with DInSAR. (Bottom) Combination of both. FISA configuration, L-band, 2003 campaign. Scene dimensions: $6.8 \mathrm{~km} \times 1.4 \mathrm{~km}$ (azimuth $\times$ slant range).

error [15]. One can estimate it using an external DEM or two corner reflectors (CRs) located at different range distances. The latter option is not possible in the 2003 campaign, considering that there was only one CR available. In the 2006 campaign, there were only two CRs; thus, calibrating with them would not allow one to evaluate the accuracy of the proposed approach. Therefore, the calibration of the constant baseline error was performed using the external DEM.

After applying the processing chain expounded in Section III-A to each group of images (see Table I), several SVFs were retrieved. Fig. 10 shows the estimated along-track and LOS displacements with the corresponding 2-D SVFs for the 2003 campaign in the FISA configuration. From the 2-D SVFs, it is clear that the along-track information is very valuable, considering that in some areas, the DInSAR measurement cannot yield any estimation due to the lack of movement in the LOS. Fig. 11 shows the same configuration as Fig. 10 but for 2006, also at L-band. The areas circled in white in the LOS displacement images of Figs. 10 and 11 show quite different estimated motions due to the fact that in the 2003 campaign, the LOS vector $\hat{\mathbf{e}}_{\text {los }}$ was almost perpendicular to the motion vector $\hat{\mathbf{e}}_{M}$, resulting in an almost zero motion measurement.

Fig. 12 shows the along-track and LOS displacements together with the 2-D SVFs of the JUJO configuration. The estimated motion in the along-track is noisier than in the FISA configuration, as the coherence is, in general, lower (see coherence analysis in the following). Finally, Fig. 13 shows the retrieved 3-D SVFs in UTM coordinates for both the L- and P-bands in the FISA configuration, obtained after applying (16).

Fig. 14 shows the one-day coherences at L-band for the 2003 and 2006 campaigns. It is interesting to observe that for FISA 2006, the (left side in the middle image) higher part of the glacier is quite coherent, in opposition to the lower part. This occurs due to melting in the lower part because of higher temperatures. The melting and coherence loss is more evident 

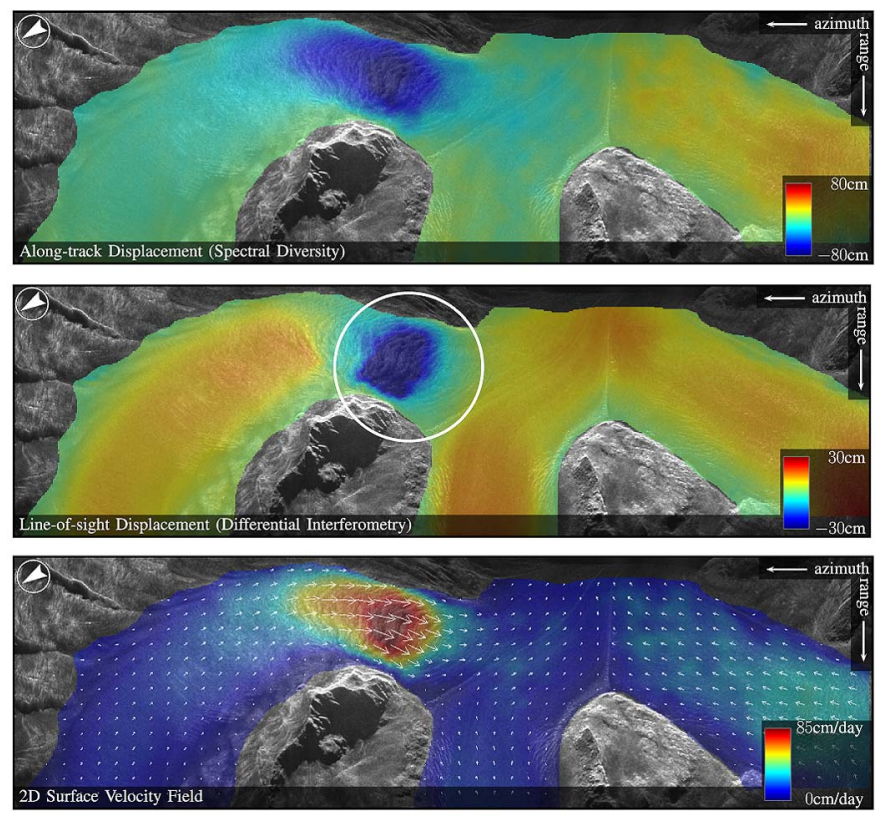

Fig. 11. Retrieval of the 2-D SVFs. (Top) Along-track displacement measured with SD. (Middle) LOS displacement measured with DInSAR. (Bottom) Combination of both. FISA configuration, L-band, 2006 campaign. Scene dimensions: $7.6 \mathrm{~km} \times 1.9 \mathrm{~km}$ (azimuth $\times$ slant-range).
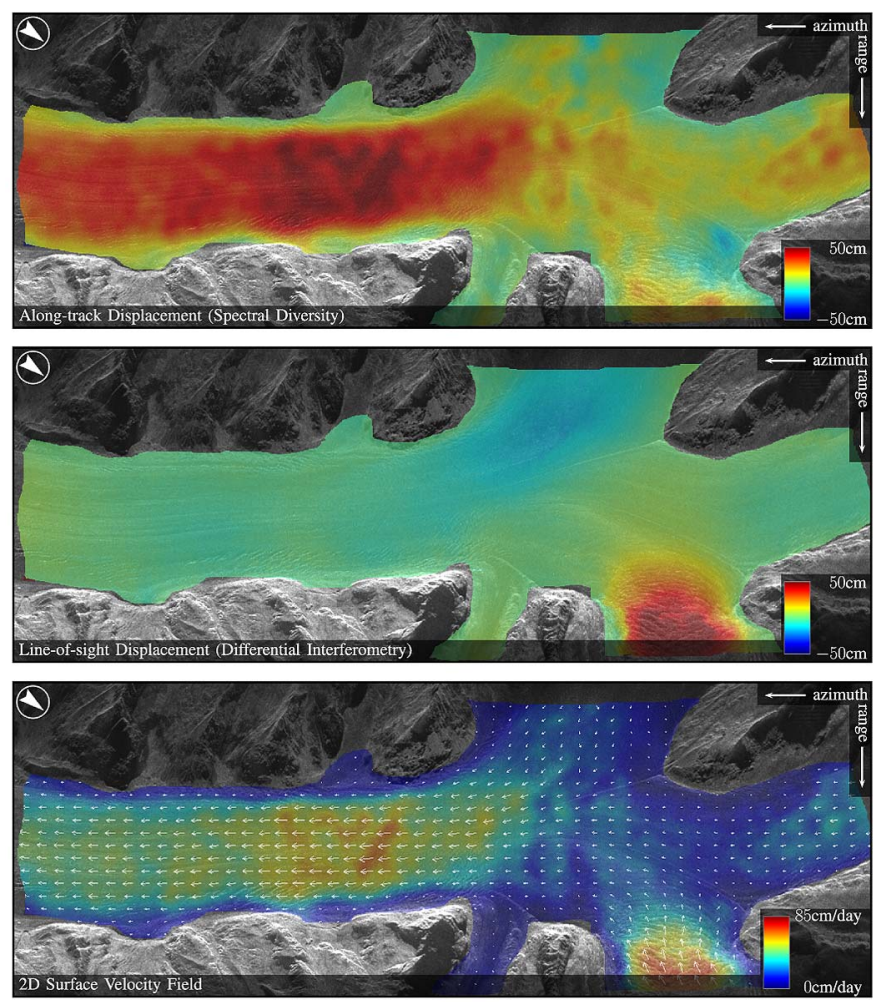

Fig. 12. Retrieval of the 2-D SVFs. (Top) Along-track displacement measured with SD. (Middle) LOS displacement measured with DInSAR. (Bottom) Combination of both. JUJO configuration, L-band, 2006 campaign. Scene dimensions: $7.5 \mathrm{~km} \times 2.3 \mathrm{~km}$ (azimuth $\times$ slant-range).

in the (bottom image) JUJO configuration. However, this is not the case for the (top image) 2003 campaign, where the coherence is kept high all over the observed portion of the glacier. For the 2006 campaign, the temperature on the science
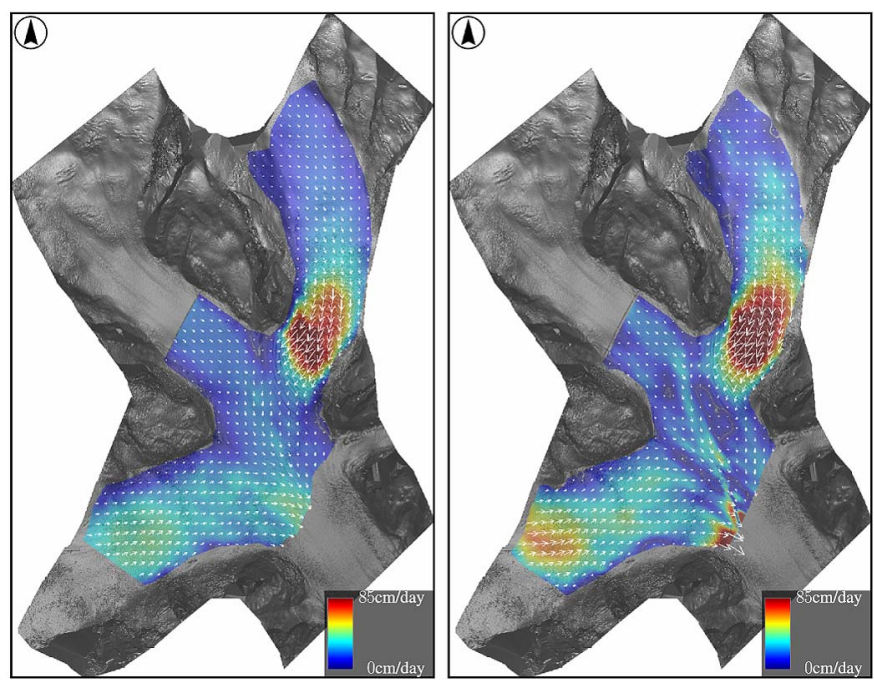

Fig. 13. Estimated SVFs in UTM coordinates derived from the 2006 acquisition in FISA configuration for (left) L- and (right) P-bands.
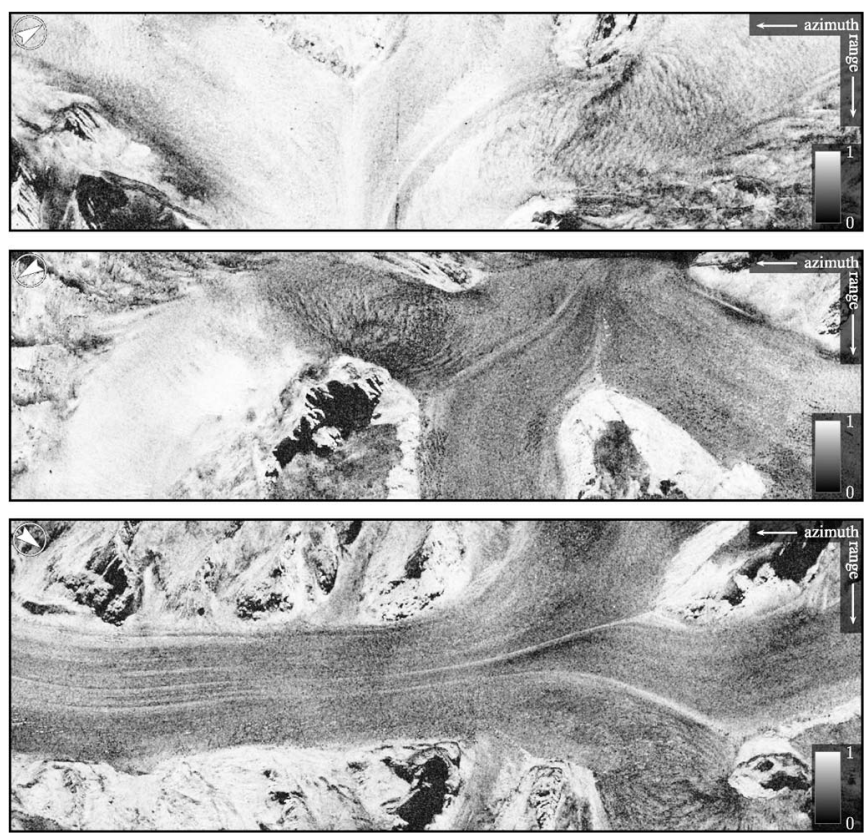

Fig. 14. Long-term coherences at L-band for (top) FISA 2003, (middle) FISA 2006, and (bottom) JUJO 2006.

observatory at JUJO (see its location in Fig. 9) was about $-3{ }^{\circ} \mathrm{C}$ in October 16 and around $0{ }^{\circ} \mathrm{C}$ in October 17 , whereas, unfortunately, no weather data are available for the 2003 campaign. The results for the 2006 campaign indicate the altitude at which temperatures start to increase above the melting point of snow within the one-day time interval of the two acquisitions. For October 16/17, 2006, this altitude was about $3200 \mathrm{~m}$. It is anticipated that frequent monitoring and coherence evaluation would allow for the estimation and tracking of the so-called equilibrium line (snow-ice boundary) [26].

The accuracy of the proposed approach can be evaluated using the positions of the CRs deployed in the scene (see location in Fig. 9), which were measured using differential GPS. For the 2003 campaign, the position of the only CR was measured on October 22 and November 5 so that a mean 

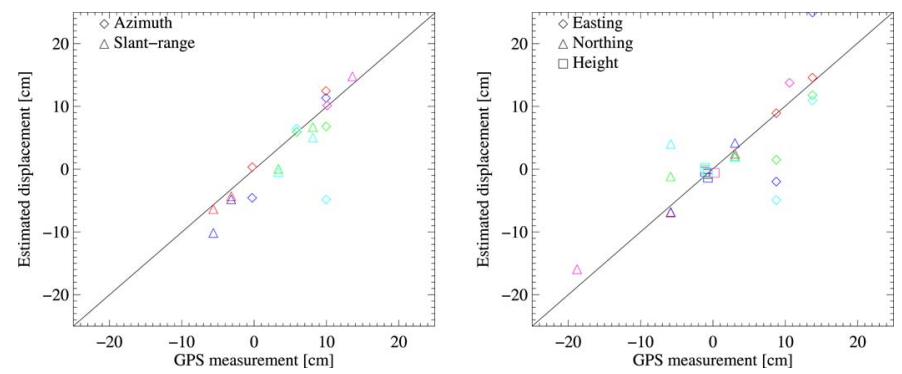

Fig. 15. Estimated motion versus GPS measurement for the CRs in the (left) azimuth/slant range and (right) UTM coordinates. The colors stand for the different configurations. (Red) FISA L-band 2006. (Green) JUJO L-band 2006. (Blue) FISA P-band 2006. (Cyan) JUJO P-band 2006. (Magenta) FISA L-band 2003.

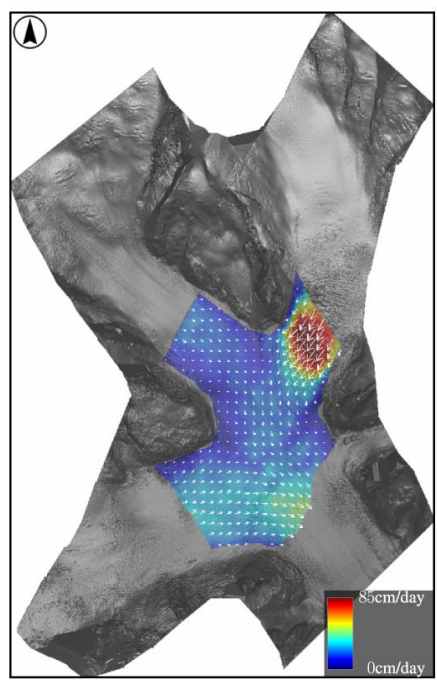

Fig. 16. Estimated SVFs at L-band in UTM coordinates derived from the combination of the FISA and JUJO configurations in 2006. In this case, the slope was not used in the estimation.

displacement was computed for the motion in centimeter per day. In the 2006 campaign, the measurements were performed within $\pm 2 \mathrm{~h}$ of the data acquisitions. The left image of Fig. 15 shows the measurements with respect to the GPS ones for each DInSAR group in the azimuth and slant-range plane, whereas the right image shows them in UTM coordinates. In general, it can be stated that the agreement with the in situ measurements is acceptable, above all, at L-band.

As commented in Section III-B, the combination of different acquisition configurations is possible. The FISA and JUJO configurations of 2006 were combined to obtain the 3-D SVFs without considering the slope, resulting in the SVFs shown in Fig. 16. The combination using only the more accurate DInSAR measurement from each configuration was also performed (note that, in this case, the slope is needed again). Fig. 17 shows the measurements in the CRs after combining the information in these two different ways. Again, results show a good agreement with the in situ measurements, and note that using the DInSAR measurement alone improves the accuracy of the result in the P-band case.

In the following, a discussion on the expected performance is presented. From the error plots, it can be stated that the accuracy at L-band is quite good and of about $1-5 \mathrm{~cm} /$ day. Nevertheless,
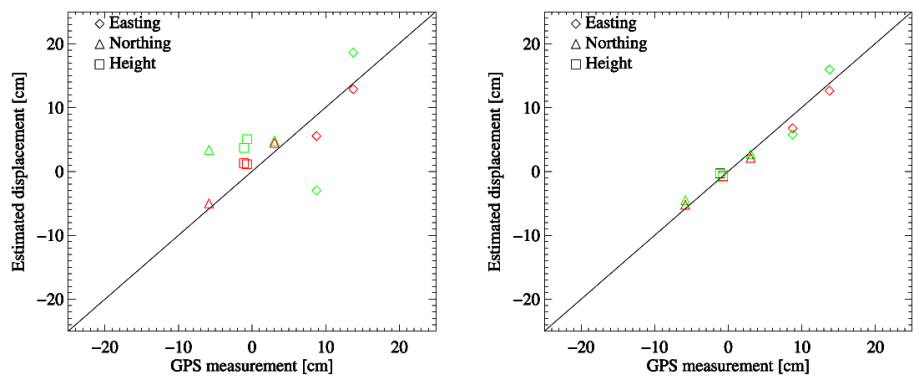

Fig. 17. Estimated motion versus GPS measurement for the CRs when combining the FISA and JUJO configurations (red) at L- and (green) P-bands, (left) when using all four available measurements (no slope used) and (right) when using only the DInSAR estimations plus the slope.

a better performance could be expected, above all, at L-band. The main effects that affect the final accuracy are as follows.

1) Data Processing: The processing of the data becomes a challenge due to the high topographic variations, which need to be considered during the focusing using an external DEM. Errors in the DEM will degrade the performance, resulting mainly in undesired coregistration (bias in the estimation of the along-track displacement of the glacier) and phase (bias in the LOS measurement) errors [24]. An iterative approach can be carried out to improve the DEM, something that could not be done at P-band due to the lack of a short-term pair. The large differences between the DEM and the P-band phase center resulted in a poor performance in this case.

2) RMEs: The second main limitation is the existence of RMEs. Using the proposed MS approach can reduce them to a large extent; however, phase ramps in azimuth and range might still persist, which can introduce phase errors as large as $1-5 \mathrm{~cm}$ at the edges of the scene. These ramps can be reduced using either an accurate DEM or a couple of CRs. Note that in the former case, the final accuracy will strongly depend on the accuracy of the external DEM.

3) Coherence: Both along-track and LOS measurements are subject to the coherence, with the former being much more sensitive (see Fig. 3). Filtering can improve the estimation to some extent, at the expense of spatial resolution.

Finally, note that just a few CRs were available during the campaign. A proper validation should consider several CRs deployed at different locations of the glacier.

\section{CONCLUSiON}

This paper has shown, for the first time, the possibility of retrieving the 2- and 3-D SVFs of a glacier using airborne SAR interferometry. In the first part of this paper, it has been shown that the main limitation of airborne repeat-pass SAR interferometry is the presence of the so-called RMEs. Usually, interferometric techniques based on the azimuth coregistration offsets between looks are used to estimate the baseline error. However, any movement of the scene itself in the along-track direction can lead to a biased estimation of the baseline error and, hence, to a degraded result. In this paper, a solution has been proposed to overcome this limitation, which makes use of the different nature of RMEs and TCE. With the EMS technique, it is possible to estimate the baseline error without being affected by the nonstationarity of the scene. 
The methodology to efficiently retrieve the SVFs using airborne SAR interferometry has been expounded next. All the steps, from SAR processing until the reconstruction of the 3-D SVFs, have been detailed. The availability of an external DEM is mandatory, considering that it is required for accurate MoCo. In the presented case, the X-band DEM acquired by the E-SAR system in the single-pass mode was used in a first iteration, whereas improved versions based on L-band data were used in further iterations. In this sense, the quality of the external DEM plays an important role, as the whole MoCo scheme is based on it.

Exemplary results have been shown with L- and P-band data acquired over the Aletsch glacier by DLR's E-SAR system, demonstrating the potential of airborne platforms to monitor SVFs. The combination of different configurations has been also shown, with the advantage that, in this case, it is not necessary to use the slope to compute the 3-D SVFs. The use of the DInSAR measurements allows for a better performance; however, in the presented results, the slope information had to be used, as only two measurements were available. Ideally, a minimum of three configurations using only the DInSAR measurements would allow an optimum estimation. This option becomes possible thanks to the flexibility in the flight configuration of airborne SAR systems.

The presented results, together with the evaluation using the CRs deployed in the scene, which resulted in an accuracy between 1-5 cm/day at L-band, allow for the validation of the proposed methodology, hence opening a new possibility to monitor temperate glaciers.

A future work will address alternative approaches to estimate the RMEs, such as the use of autofocus techniques using isolated or pointlike scatterers [27], [28]. Moreover, the analysis of the derived SVFs as input to glacier flow models will be the subject of future studies.

\section{ACKNOWLEDGMENT}

The authors would like to thank the Remote Sensing Laboratories team of University of Zürich for campaign preparations and ground measurements and DLR's E-SAR team for the flights. The SWISAR campaign was performed with the funding of "armasuisse" Switzerland. The authors would also like to thank the anonymous reviewers for their comments and suggestions.

\section{REFERENCES}

[1] M. Lüthi and M. Funk, "Dating ice cores from a high alpine glacier with a flow model for cold firn," Ann. Glaciol., vol. 31, no. 1, pp. 69-79, Jan. 2000.

[2] R. M. Goldstein, H. Engelhardt, B. Kamb, and R. M. Frolich, "Satellite radar interferometry for monitoring ice sheet motion: Application to an Antarctic ice stream," Science, vol. 262, no. 5139, pp. 1525-1530, Dec. 1993.

[3] R. Kwok and M. Fahnestock, "Ice sheet motion and topography from radar interferometry," IEEE Trans. Geosci. Remote Sens., vol. 34, no. 1, pp. 189-220, Jan. 1996.

[4] I. R. Joughin, R. Kwok, and M. A. Fahnestock, "Interferometric estimation of three-dimensional ice-flow using ascending and descending passes," IEEE Trans. Geosci. Remote Sens., vol. 36, no. 1, pp. 25-37, Jan. 1998
[5] T. Strozzi, A. Luckman, T. Murray, U. Wegmüller, and C. L. Werner, "Glacier motion estimation using SAR offset-tracking procedures," IEEE Trans. Geosci. Remote Sens., vol. 40, no. 11, pp. 2384-2391, Nov. 2002.

[6] E. Trouvé, G. Vasile, M. Gay, L. Bombrun, P. Grussenmeyer, T. Landes, J.-M. Nicolas, P. Bolon, I. Petillot, A. Julea, L. Valet, J. Chanussot, and M. Koehl, "Combining airborne photographs and spaceborne SAR data to monitor temperate glaciers: Potentials and limits," IEEE Trans. Geosci. Remote Sens., vol. 45, no. 4, pp. 905-924, Apr. 2007.

[7] T. Strozzi, U. Wegmüller, C. Werner, and A. Wiesmann, "Surface velocity of Swiss alpine glaciers from ERS SAR interferometry," in Proc. ENVISAT Symp., Montreux, Switzerland, Apr. 23-27, 2007.

[8] E. Erten, A. Reigber, and O. Hellwich, "Generation of three-dimensional deformation map at low resolution using a combination of spectral diversity via least square approach," in Proc. ENVISAT Symp., Montreux, Switzerland, Apr. 23-27, 2007.

[9] A. Moreira, J. Mittermayer, and R. Scheiber, "Extended chirp scaling algorithm for air- and spaceborne SAR data processing in stripmap and ScanSAR imaging modes," IEEE Trans. Geosci. Remote Sens., vol. 34 , no. 5, pp. 1123-1136, Sep. 1996.

[10] K. A. C. de Macedo and R. Scheiber, "Precise topography- and aperturedependent motion compensation for airborne SAR," IEEE Geosci. Remote Sens. Lett., vol. 2, no. 2, pp. 172-176, Apr. 2005.

[11] P. Prats, A. Reigber, and J. J. Mallorqui, "Topography-dependent motion compensation for repeat-pass interferometric SAR systems," IEEE Geosci. Remote Sens. Lett., vol. 2, no. 2, pp. 206-210, Apr. 2005.

[12] R. J. Bullock, R. Voles, A. Currie, H. D. Griffiths, and P. V. Brennan, "Estimation and correction of roll errors in dual antenna interferometric SAR," in Proc. IEE Radar, Edinburgh, U.K., Oct. 14-16, 1997, pp. $253-257$.

[13] A. Reigber, "Correction of residual motion errors in airborne SAR interferometry," Electron. Lett., vol. 37, no. 17, pp. 1083-1084, Aug. 2001.

[14] P. Prats and J. J. Mallorqui, "Estimation of azimuth phase undulations with multisquint processing in airborne interferometric SAR images," IEEE Trans. Geosci. Remote Sens., vol. 41, no. 6, pp. 1530-1533, Jun. 2003.

[15] A. Reigber, P. Prats, and J. J. Mallorqui, "Refined estimation of timevarying baseline errors in airborne SAR interferometry," IEEE Trans. Geosci. Remote Sens., vol. 3, no. 1, pp. 145-149, Jan. 2006.

[16] R. Scheiber and A. Moreira, "Coregistration of interferometric SAR images using spectral diversity," IEEE Trans. Geosci. Remote Sens., vol. 38 , no. 5, pp. 2179-2191, Jul. 2000.

[17] G. Fornaro, "Trajectory deviations in airborne SAR: Analysis and compensation," IEEE Trans. Aerosp. Electron. Syst., vol. 35, no. 3, pp. 997-1009, Jul. 1999.

[18] R. Bamler and M. Eineder, "Accuracy of differential shift estimation by correlation and split-bandwidth interferometry for wideband and Delta-k SAR systems," IEEE Geosci. Remote Sens. Lett., vol. 2, no. 2, pp. 151-155, Apr. 2005.

[19] P. Prats, C. Andres, R. Scheiber, K. A. C. de Macedo, J. Fischer, and A. Reigber, "Glacier displacement field estimation using airborne SAR interferometry," in Proc. IEEE IGARSS, Barcelona, Spain, Jul. 23-27, 2007, pp. 2098-2101.

[20] A. K. Gabriel, R. M. Goldstein, and H. A. Zebker, "Mapping small elevation changes over large areas: Differential radar interferometry," J. Geophys. Res., vol. 94, no. B7, pp. 9183-9191, 1989.

[21] D. Massonnet, M. Rossi, C. Carmona, F. Adragna, G. Peltzer, K. Fiegl, and T. Rabaute, "The displacement field of the Landers earthquake mapped by radar interferometry," Nature, vol. 364 , no. 6433, pp. 138 142, Jul. 1993

[22] L. M. H. Ulander, H. Hellsten, and G. Stenström, "Synthetic aperture radar processing using fast factorized back-projection," IEEE Trans. Aerosp. Electron. Syst., vol. 39, no. 3, pp. 760-776, Jul. 2003.

[23] P. Prats, K. A. C. de Macedo, A. Reigber, R. Scheiber, and J. J. Mallorqui, "Comparison of topography- and aperture dependent motion compensation algorithms for airborne SAR," IEEE Geosci. Remote Sens. Lett., vol. 4, no. 3, pp. 349-353, Jul. 2007.

[24] P. Prats, A. Reigber, J. J. Mallorqui, R. Scheiber, and A. Moreira, "Estimation of the temporal evolution of the deformation using airborne differential SAR interferometry," IEEE Trans. Geosci. Remote Sens., vol. 46, no. 4, pp. 1065-1078, Apr. 2008.

[25] E. Rodriguez and J. M. Martin, "Theory and design of interferometric synthetic aperture radars," Proc. Inst. Elect. Eng.-F, vol. 139, no. 2, pp. 147-159, Apr. 1992.

[26] J. Schaper, H. Rehrauer, and K. Seidel, "Determination of the equilibrium line on an alpine glacier using remote sensing data fusion techniques," in Proc. IEEE IGARSS, Seattle, WA, Jul. 6-10, 1998, pp. 1855-1857. 
[27] H. Cantalloube and P. Dubois-Fernandez, "Airborne X-band SAR imaging with $10 \mathrm{~cm}$ resolution: Technical challenge and preliminary results," Proc. Inst. Elect. Eng.-Radar, Sonar Navig., vol. 153, no. 2, pp. 163-176, Apr. 2006.

[28] K. A. C. de Macedo, R. Scheiber, and A. Moreira, "An autofocus approach for residual motion errors with application to airborne repeat-pass SAR interferometry," IEEE Trans. Geosci. Remote Sens., vol. 46, no. 10, Oct. 2008 , to be published.

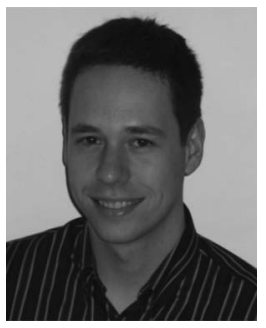

Pau Prats (S'03-M’06) was born in Madrid, Spain, in 1977. He received the Ingeniero degree in telecommunication engineering and the Ph.D. degree from the Universitat Politècnica de Catalunya (UPC), Barcelona, Spain, in 2001 and 2006, respectively.

In 2001, he was with the Institute of Geomatics, Spain, as a Research Assistant designing a subaperture SAR processor. In 2002, he was with the Department of Signal Theory and Communications, UPC, where he worked in the field of airborne repeatpass interferometry and airborne differential SAR interferometry. From December 2002 to August 2006, he was an Assistant Professor with the Department of Telecommunications and Systems Engineering, Universitat Autònoma de Barcelona, Barcelona, Spain. Since August 2006, he has been with the SAR Processing Group, Microwaves and Radar Institue, German Aerospace Center, Oberpfaffenhofen, Germany, as a Research Scientist working in the field of SAR processing and interferometry. His research interests include SAR processing, airborne motion compensation, single/repeatpass interferometry, and differential interferometry with airborne systems.

Dr. Prats was the recipient of the first prize of the Student Paper Competition of the IEEE International Geoscience and Remote Sensing Symposium 2005 Conference held in Seoul, Korea.

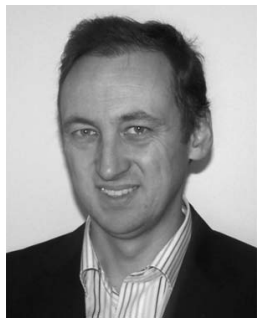

Rolf Scheiber received the Diploma degree in electrical engineering from the Technical University of Munich, Munich, Germany, in 1994 and the Ph.D. degree in electrical engineering from the University of Karlsruhe, Karlsruhe, Germany, in 2003, with a thesis on airborne SAR interferometry.

Since 1994, he has been with the Microwaves and Radar Institute, German Aerospace Center, Oberpfaffenhofen, Germany, where he developed the operational high precision interferometric SAR processor for its E-SAR airborne sensor and where since 2001, he has been heading the SAR signal processing group within the SAR Technology Department and is currently responsible for the E-SAR campaign data processing, as well as the development of the processing software for the new airborne sensor F-SAR. His current research interests include algorithm development for high-resolution air- and spaceborne SAR focusing, SAR interferometry, differential SAR interferometry, SAR tomography, as well as radio sounding algorithms and applications.

In 1997, Dr. Scheiber was the recipient of the 1996 IEEE Geoscience and Remote Sensing Society Transactions Prize Paper Award for the contribution "Extended Chirp Scaling Algorithm for Air- and Spaceborne SAR Data Processing in Stripmap and ScanSAR Imaging Modes," of which he was a coauthor.

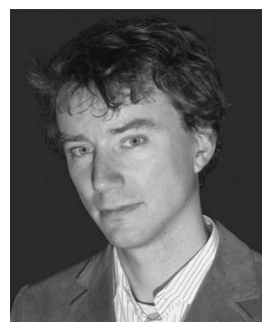

Andreas Reigber (M'02) was born in Munich, Germany, in 1970. He received the Diploma degree in physics from the University of Constance, Konstanz, Germany, in 1997, the Ph.D. degree from the University of Stuttgart, Stuttgart, Germany, in 2001, and a habilitation from the Berlin University of Technology, Berlin, Germany, in 2008

From 1996 to 2000, he was with the Microwaves and Radar Institute, German Aerospace Center (DLR), Oberpfaffenhofen, Germany, working in the field of polarimetric SAR tomography. In 2001, he was with the Antenna, Radar, and Telecom Laboratories, University of Rennes 1, Rennes, France, for a Postdoctoral Fellowship on radar polarimetry and polarimetric interferometry. From 2002 to 2007, he was a Research Associate with the Computer Vision and Remote Sensing Laboratories, Berlin University of Technology, Berlin. Since 2008, he has been with the Microwaves and Radar Institute, DLR, where he is currently heading the SAR Technology Department. His current main research interests are the various aspects of multimodal SAR, like-SAR interferometry, SAR polarimetry, SAR tomography, and time-frequency analyses, as well as hyperspectral remote sensing and the application of computer vision and machine learning approaches in remote sensing.

Dr. Reigber was the recipient of the EUSAR 2000 Student Prize Paper Award for an article on SAR remote sensing of forests, the IEEE TRANSACTIONS on Geoscience and Remote Sensing (TGRS) Transactions Prize Paper Award in 2001 for a work on polarimetric SAR tomography, and the IEEE TGRS Letters Prize Paper Award in 2006 for a work on multipass SAR processing. He also coauthored three papers which have been successful at the student paper competitions of the IEEE International Geoscience and Remote Sensing Symposium (IGARSS)'05 (first prize), EUSAR'06 (third prize), and IGARSS'07 (second prize).

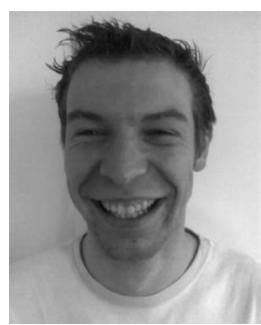

Christian Andres received the Dipl. degree in information technology from the University of Applied Sciences Wiener Neustadt, Wiener Neustadt, Austria, in 2005.

In 2004, he was with the SAR Technology Department, Microwaves and Radar Institute, German Aerospace Center, Wessling, Germany, for the development of SAR signal processing algorithms for the experimental airborne SAR sensor (E-SAR) and its successor F-SAR. His research interests include airborne SAR interferometry, geocoding, motion compensation, and differential SAR interferometry.

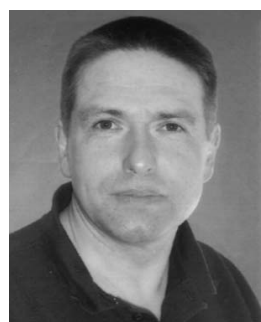

Ralf Horn received the Dipl.-Ing. degree in electrical engineering, with major subjects in telecommunications and radio-frequency engineering, from Ruhr Universitaet Bochum, Bochum, Germany, in 1983.

Since 1983, he has been with the SAR Technology Department, Microwaves and Radar Institute, German Aerospace Center (DLR), Oberpfaffenhofen, Germany, where from 1983 to 1986, he was first a Radar Engineer for the development of DLR's airborne Experimental SAR system E-SAR and was later a SAR Systems Engineer; then, in 1994, he was promoted to Team Leader of the "Airborne SAR" group of the SAR Technology Department. His current responsibilities are focused on the project management for the new DLR airborne SAR system F-SAR and on the management, coordination, and execution of the DLR airborne SAR missions in Europe and abroad. 